doi: https://doi.org/10.15407/dopovidi2019.03.017

UDC 517.5

V.Ya. Gutlyanskii ${ }^{1}$, V.I. Ryazanov ${ }^{1,2}$, E. Yakubov ${ }^{3}$, A.S. Yefimushkin ${ }^{1}$

${ }^{1}$ Institute of Applied Mathematics and Mechanics of the NAS of Ukraine, Slov'yansk

${ }^{2}$ Bogdan Khmelnytsky National University of Cherkasy

${ }^{3}$ Holon Institute of Technology, Israel

E-mail: vgutlyanskii@gmail.com,vl.ryazanov1@gmail.com, yakubov@hit.ac.il, eduardyakubov@gmail.com, a.yefimushkin@gmail.com

\title{
On boundary-value problems in domains without (A)-condition
}

Presented by Corresponding Member of the NAS of Ukraine V.Ya. Gutlyanskii

We study the Hilbert boundary-value problem for the Beltrami equations in the Jordan domains satisfying the quasihyperbolic boundary condition by Gehring-Martio, generally speaking, without the standard (A)-condition by Ladyzhenskaya-Ural'tseva. Assuming that the coefficients of the problem are functions of countable bounded variation and the boundary data are measurable with respect to the logarithmic capacity, we prove the existence of its solutions. As consequences, we derive the existence of nonclassical solutions of the Dirichlet, Neumann and Poincaré boundary-value problems for generalizations of the Laplace equation in anisotropic and inhomogeneous media.

Keywords: Hilbert, Dirichlet, Neumann, and Poincaré boundary-value problems, Beltrami equations, quasiconformal functions, angular limits, quasihyperbolic boundary condition, logarithmic capacity.

1. Introduction. Let $D$ be a domain in the complex plane $\mathbb{C}$, and let $\mu: D \rightarrow \mathbb{C}$ be a measurable function with $|\mu(z)|<1$ a.e. The equation of the form

$$
f_{\bar{z}}=\mu(z) f_{z},
$$

where $f_{\bar{z}}=\bar{\partial} f=\left(f_{x}+i f_{y}\right) / 2, f_{z}=\partial f=\left(f_{x}-i f_{y}\right) / 2, z=x+i y, f_{x}$ and $f_{y}$ are partial derivatives of $f$ with respect to $x$ and $y$, is said to be a Beltrami equation. Equation (1) is called nondegenerate if $\|\mu\|_{\infty}<1$. Homeomorphic solutions $f$ of the nondegenerate equation (1) in the class $W_{\text {loc }}^{1,2}$ are called quasiconformal mappings (see, e.g., [1]).

D. Hilbert studied the boundary-value problem formulated as follows: To find an analytic function $f(z)$ in a domain $D$ bounded by a rectifiable Jordan contour $C$ that satisfies the boundary condition

$$
\lim _{z \rightarrow \zeta} \operatorname{Re}\{\overline{\lambda(\zeta)} f(z)\}=\varphi(\zeta) \quad \forall \zeta \in C,
$$

(C) V.Ya. Gutlyanskii, V.I. Ryazanov, E. Yakubov, A.S. Yefimushkin, 2019 
where both the coefficient $\lambda$ and the boundary data $\varphi$ of the problem are continuously differentiable with respect to the natural parameter $s$ on $C$. Moreover, it was assumed by Hilbert that $\lambda \neq 0$ everywhere on $C$.

The latter allows us with no loss of generality to consider that $|\lambda| \equiv 1$ on $C$. In this case, the quantity $\operatorname{Re}\{\bar{\lambda} f\}$ from the left in (2) has the geometric sense as a projection of $f$ onto the direction $\lambda$ interpreted as vectors in $\mathbb{R}^{2}$.

Historic comments in the subject can be found in the recent paper [2]. In the present paper, we study the Hilbert boundary-value problem for the Beltrami equation and find its regular solutions in the class of quasiconformal functions $F$ represented as a composition of analytic functions $A$ and quasiconformal mappings $f$ satisfying (2).

Proceeding the above, the problem under consideration is to find quasiconformal functions satisfying both the Beltrami equation (1) in a Jordan domain $D$ and the Hilbert boundary condition (2). We substantially weaken the regularity conditions both on the functions $\lambda$ and $\varphi$ in the boundary condition (2) and on the boundary $C$ of the domain $D$. On the one hand, we will deal with the coefficients $\lambda$ of a countable bounded variation and the boundary data $\varphi$, which are measurable with respect to the logarithmic capacity, see the corresponding definitions in our previous paper [3]. On the other hand, we study here the Hilbert boundary-value problem in domains $D$ with a more general boundary condition, see discussions in the next section.

2. On the quasihyperbolic boundary condition. Let $D$ be a domain in $\mathbb{C}$. As usual, $k_{D}\left(z, z_{0}\right)$ denotes the quasihyperbolic distance between points $z$ and $z_{0}$ in $D$

$$
k_{D}\left(z, z_{0}\right)=\inf _{\gamma} \int_{\gamma} \frac{d s}{d(\varsigma, \partial D)}
$$

introduced in work [4]. Here, $d(\zeta, \partial D)$ denotes the Euclidean distance from the point $\zeta \in D$ to $\partial D$, and the infimum is taken over all rectifiable curves $\gamma$ joining the points $z$ and $z_{0}$ in $D$.

Further, it is said that a domain $D$ satisfies the quasihyperbolic boundary condition if

$$
k_{D}\left(z, z_{0}\right) \leqslant a \ln \frac{d\left(z_{0}, \partial D\right)}{d(z, \partial D)}+b \quad \forall z \in D
$$

for constants $a$ and $b$ and a point $z_{0} \in D$. The latter notion was introduced in [5], but it was first applied in [6].

Recall also that the images of the unit disk $\mathbb{D}=\{z \in \mathbb{C}:|z|<1\}$ under the quasiconformal mappings of $\mathbb{C}$ onto itself are called quasidisks, and their boundaries are called quasicircles or quasiconformal curves. It is known that every smooth (or Lipschitz) Jordan curve is a quasiconformal curve, and, at the same time, quasiconformal curves can be locally nonrectifiable, as it follows from the known examples (see, e.g., point II.8.10 in [1]).

Remark 1. Quasidisks $D$ satisfy the quasihyperbolic boundary condition. Indeed, as is well known, the Riemann conformal mapping $\omega: D \rightarrow \mathbb{D}$ is extended to a quasiconformal mapping of $\mathbb{C}$ onto itself (see, e.g., Theorem II.8.3 in [1]). By one of the main Bojarski results, the derivatives of a quasiconformal mapping in the plane are locally integrable with some power $q>2$, and its Jacobian $J(w)=\left|\omega_{w}\right|^{2}-\left|\omega_{\bar{\psi}}\right|^{2}$ (see [1]). Consequently, $J \in L^{p}(D)$ in this case for some $p>1$, and we have the desired conclusion by the criterion in Theorem 2.4 [7]. 
Recall that a domain $D$ in $\mathbb{R}^{n}, n \geqslant 2$, is called satisfying $(A)$-condition if

mes $D \cap B(\zeta, \rho) \leqslant \Theta_{0}$ mes $B(\zeta, \rho) \quad \forall \zeta \in \partial D, \quad \rho \leqslant \rho_{0}$

for some $\Theta_{0}$ and $\rho_{0} \in(0,1)$ (see 1.1.3 in [8]). Recall also that a domain $D$ in $\mathbb{R}^{n}, n \geqslant 2$, is said to be satisfying the outer cone condition if there is a cone that makes possible to be touched by its top to every boundary point of $D$ from the completion of $D$ after its suitable rotations and shifts. It is clear that the outer cone condition implies (A)-condition. It is well known that the above conditions are standard in the theory of boundary-value problems for the partial differential equations.

Remark 2. Note that quasidisks $D$ satisfy (A)-condition. Indeed, the quasidisks are the socalled $Q E D$ - domains by Gehring-Martio (see Theorem 2.22 in [9]), and the latter satisfy the condition

$$
\text { mes } D \cap B(\zeta, \rho) \geqslant \Theta_{*} \operatorname{mes} B(\zeta, \rho) \quad \forall \zeta \in \partial D, \quad \rho \leqslant \operatorname{diam} D
$$

for some $\Theta_{*} \in(0,1)$ (see Lemma 2.13 in [9]), and quasidisks (as domains with quasihyperbolic boundary condition) have boundaries of the Lebesgue measure zero (see, e.g., Theorem 2.4 in [7]). Thus, it remains to note that, by definition, the completions of quasidisks $D$ in the extended complex plane $\overline{\mathbb{C}}:=\mathbb{C} \cup\{\infty\}$ are also quasidisks up to the inversion with respect to a circle in $D$. As we know, the first example of a simply connected plane domain $D$ with the quasihyperbolic boundary condition, which is not a quasidisk, was constructed in Theorem 2 [6]. However, this domain satisfied (A)-condition.

Remark 3. Probably one of the simplest examples of a domain $D$ with the quasihyperbolic boundary condition and without (A)-condition is the union of 3 open disks with radius 1 centered at the points 0 and $1 \pm i$. It is clear that the domain has zero interior angle at its boundary point 1 , and, by Remark 2 , it is not a quasidisk. Note that $\partial D$ is almost smooth. Thus, there exist almost smooth Jordan curves (see (5) in [3]) with the quasihyperbolic boundary condition that are not quasiconformal curves.

3. The Hilbert problem for the Beltrami equation. Let $D$ be a Jordan domain with a tangent at a point $\varsigma \in \partial D$. A path in $D$ terminating at $\zeta$ is called nontangential if its part in a neighborhood of $\zeta$ lies inside of an angle in $D$ with the vertex at $\zeta$. The limit along all nontangential paths at $\zeta$ is called angular at the point. The latter notion is a standard tool for the study of the boundary behavior of analytic and harmonic functions (see, e.g., [10]).

Theorem 1. Let $D$ be a Jordan domain with the quasihyperbolic boundary condition, and let $\partial D$ have a tangent q.e. Suppose that $\mu: D \rightarrow \mathbb{C}$ is in $L^{\infty}(D)$ with $\|\mu\|_{\infty}<1, \lambda: \partial D \rightarrow \mathbb{C},|\lambda(\zeta)| \equiv 1$, is in $\mathcal{C B V}(\partial D)$, and $\varphi: \partial D \rightarrow \mathbb{R}$ is a measurable function with respect to the logarithmic capacity. Then the Beltrami equation (1) has a regular solution $f: D \rightarrow \mathbb{C}$ with the angular limit

$$
\lim _{z \rightarrow \zeta} \operatorname{Re}[\overline{\lambda(\zeta)} f(z)]=\varphi(\zeta) \text { q.e. on } \partial D .
$$

Proof. Let $g$ be a conformal mapping of $D$ onto $\mathbb{D}$ that exists by the Riemann mapping theorem (see, e.g., Theorem II.2.1 in [11]). Setting, in the unit disk $\mathbb{D}$,

$$
v(w):=\left(\mu \frac{g^{\prime}}{\bar{g}^{\prime}}\right) \circ g^{-1}(w),
$$


we see that $v \in L^{\infty}(\mathbb{D})$ and $\|v\|_{\infty}=\|\mu\|_{\infty}<1$. Hence, by the Measurable Riemann Mapping theorem (see, e.g., [1]), there is a quasiconformal mapping $G$ of $\mathbb{D}$ onto itself, $G(0)=0$, satisfying the Beltrami equation $G_{\bar{w}}=v(w) G_{w}$ a.e. in $\mathbb{D}$. By the reflection principle (see, e.g., Theorem I.8.4 in [1]), $G$ can be extended to a quasiconformal mapping $\tilde{G}$ of $\mathbb{C}$ onto itself. Both functions $G_{*}:=\left.\tilde{G}\right|_{\partial \mathbb{D}}$ and $G_{*}^{-1}$ are Hölder continuous (see, e.g., [1], Theorem II.4.3). Now, by the Carathéodory theorem (see, e.g., Theorem II.3.4 in [11]), $g$ is extended to a homeomorphism $\tilde{g}$ of $\bar{D}$ onto $\overline{\mathbb{D}}$. By Corollary of Theorem 1 in [6], $g_{*}:=\left.\tilde{g}\right|_{\partial D}$ and its inverse function are Hölder continuous. Thus, the mapping $h_{*}:=G_{*} \circ g_{*}: \partial D \rightarrow \partial \mathbb{D}$ and its inverse are also Hölder continuous. In particular, then $\Lambda:=\lambda \circ h_{*}^{-1} \in \mathcal{C B V}(\partial \mathbb{D})$ and $\Phi:=\varphi \circ h_{*}^{-1}$ is measurable with respect to the logarithmic capacity by Remark 1 in [3]. Next, by Theorem 1 in [3], there is an analytic function $A: \mathbb{D} \rightarrow \mathbb{C}$ that has the angular limit

$$
\lim _{\omega \rightarrow \eta} \operatorname{Re}\{\overline{\Lambda(\eta)} A(\omega)\}=\Phi(\eta) \text { q.e. on } \partial \mathbb{D} .
$$

Setting $h:=G \circ g$, we see by an elementary computation that $h_{z}=G_{w} \circ g(z) g^{\prime}(z)$ and $h_{z}=$ $G_{\varpi} \circ g(z) \overline{g^{\prime}(z)}$ a.e. in $D$, i.e. $h$ is a quasiconformal mapping of $D$ onto $\mathbb{D}$ satisfying Eq. (1) a.e. in $D$.

Let us consider the function $f:=A \circ h$. Since $f_{z}=A^{\prime} \circ h(z) h_{z}$ and $f_{\bar{z}}=A^{\prime} \circ h(z) h_{\bar{z}}$ a.e. in $D$, we see that $f$ satisfies the Eq. (1). On the other hand, the mapping $f$ is continuous, open, and discrete, and, therefore, $f$ is the regular solution of (1). It remains to show that $f$ satisfies also the boundary condition (2). Indeed, by the Lindelöf theorem (see, e.g., Theorem II.C.2 in [10]), if $\partial D$ has a tangent at a point $\zeta$, then $\arg [g(\zeta)-g(z)]-\arg [\zeta-z] \rightarrow$ const as $z \rightarrow \zeta$. In other words, the images under the conformal mapping $g$ of sectors in $D$ with a vertex at $\zeta$ are asymptotically the same as sectors in $\mathbb{D}$ with a vertex at $w=g(\zeta)$. Consequently, nontangential paths in $D$ are transformed under $g$ into nontangential paths in $\mathbb{D}$ and inversely q.e. on $\partial D$ and $\partial \mathbb{D}$ respectively, because $D$ is almost smooth, and $g_{*}$ and $g_{*}^{-1}$ keep sets of logarithmic capacity zero. Moreover, it is known that the distortion of angles under a quasiconformal mapping is bounded (see, e.g., [1]). Hence, the mapping $\tilde{G}: \mathbb{C} \rightarrow \mathbb{C}$ and its inverse also transform nontangential paths into nontangential paths, and $G_{*}$ and $G_{*}^{-1}$ keep sets of logarithmic capacity zero. Consequently, $h: D \rightarrow \mathbb{D}$ and $h^{-1}: \mathbb{D} \rightarrow D$ also transform nontangential paths into nontangential paths q.e. on $\partial D$ and $\partial \mathbb{D}$ respectively. Thus, (9) implies the existence of the angular limit (2) q.e. on $\partial D$.

4. On Dirichlet, Neumann, and Poincaré problems. Recall that (see, e.g., Theorem 16.1.6 in [12]) if $f=u+i v$ is a regular solution of the nondegenerate Beltrami equation (1), then the function $u$ is a continuous generalized solution of the divergence type equation

$$
\operatorname{div} A(z) \nabla u=0
$$

and is called the $A$-harmonic function, where $A(z)$ is a matrix function:

$$
A(z)=\left(\begin{array}{ll}
\frac{|1-\mu(z)|^{2}}{1-|\mu(z)|^{2}} & \frac{-2 \operatorname{Im} \mu(z)}{1-|\mu(z)|^{2}} \\
\frac{-2 \operatorname{Im} \mu(z)}{1-|\mu(z)|^{2}} & \frac{|1+\mu(z)|^{2}}{1-|\mu(z)|^{2}}
\end{array}\right) .
$$


As we see, the matrix function $A(z)$ in (11) is symmetric, $\operatorname{det} A(z) \equiv 1$, and its entries $a_{i j}=a_{i j}(z)$ are dominated by the quantity $K_{\mu}(z)=\frac{1+|\mu(z)|}{1-|\mu(z)|}$. Thus, they are bounded if Beltrami's equation (1) is not degenerate. Vice versa, the uniformly elliptic equations (10) with symmetric $A(z)$ and $\operatorname{det} A(z) \equiv 1$ just correspond to the nondegenerate Beltrami equations (1) with the coefficient

$$
\mu=\frac{1}{\operatorname{det}(I+A)}\left(a_{22}-a_{11}-2 i a_{21}\right)=\frac{a_{22}-a_{11}-2 i a_{21}}{1+\operatorname{Tr} A+\operatorname{det} A} .
$$

We denote by $\mathcal{B}$ the collection of all such matrix functions $A(z)$ Recall that Eq. (10) is said to be uniformly elliptic if $a_{i j} \in L^{\infty}$ and $\langle A(z) \eta, \eta\rangle \geqslant \varepsilon|\eta|^{2}$ for some $\varepsilon>0$ and for all $\eta \in \mathbb{R}^{2}$.

Corollary 1. Let $D$ be a domain in $\mathbb{C}$ with the quasihyperbolic boundary condition, and let $\partial D$ have a tangent q. e. Suppose that $A \in \mathcal{B}$ and $\varphi: \partial D \rightarrow \mathbb{R}$ is measurable with respect to the logarithmic capacity. Then there exists an A-harmonic function $u: D \rightarrow \mathbb{R}$ with the angular limit

$$
\lim _{\omega \rightarrow \eta} u(z)=\varphi(\zeta) \text { q.e. on } \partial D \text {. }
$$

Theorem 2. Let $D$ be a domain in $\mathbb{C}$ with the quasihyperbolic boundary condition, and let $\partial D$ have a tangent q. e. Suppose that $A(z), z \in D$, is a matrix function in the class $\mathcal{B} \cap C^{\alpha}, \alpha \in(0,1)$, $v: \partial D \rightarrow \mathbb{C},|v(\zeta)| \equiv 1$, is in the class $\mathcal{C B V}$ and $\varphi: \partial D \rightarrow \mathbb{R}$ is measurable with respect to logarithmic capacity. Then there exists an $A$-harmonic function $u: D \rightarrow \mathbb{R}$ in the class $C^{1+\alpha}$ with the angular limit

$$
\lim _{z \rightarrow \varsigma} \frac{\partial u}{\partial v}(z)=\varphi(\zeta) \text { q.e. on } \partial D \text {. }
$$

Proof. By the above remarks, the desired function $u$ is the real part of a solution $f$ in the class $W_{\text {loc }}^{1,1}$ for the Beltrami equation (1) with $\mu \in C^{\alpha}$ given by formula (12). By Lemma 1 in [13], $\mu$ is extended to a Hölder continuous function $\mu_{*}: \mathbb{C} \rightarrow \mathbb{C}$ of the class $C^{\alpha}$. Set $k=\max |\mu(z)|<1$ in $D$. Then, for every $k_{*} \in(k, 1)$, there is an open neighborhood $U$ of $D$, where $\left|\mu_{*}(z)\right| \leqslant k_{*}$. Let $D_{*}$ be a connected component of $U$ containing $\bar{D}$.

By the Measurable Riemann Mapping Theorem (see, e.g., [1]), there is a quasiconformal mapping $h: D_{*} \rightarrow \mathbb{C}$ a.e. satisfying the Beltrami equation (1) with the complex coefficient $\mu^{*}:=\left.\mu_{*}\right|_{D_{*}}$ in $D_{*}$. Note that the mapping $h$ has the Hölder continuous first partial derivatives in $D_{*}$ with the same order of the Hölder continuity as $\mu$ (see, e.g., [14]). Moreover, its Jacobian

$$
J_{h}(z) \neq 0 \quad \forall z \in D_{*},
$$

(see, e.g., Theorem V.7.1 in [1]). Thus, the directional derivative

$$
h_{\omega}(z)=\frac{\partial h}{\partial \omega}(z):=\lim _{t \rightarrow 0} \frac{h(z+t \omega)-h(z)}{t} \neq 0 \quad \forall z \in D_{*} \forall \omega \in \partial \mathbb{D},
$$

and it is continuous in the collection of the variables $\omega \in \partial \mathbb{D}$ and $z \in D_{*}$. Thus, the functions

$$
\nu_{*}(\zeta):=\frac{\left|h_{v(\zeta)}(\zeta)\right|}{h_{v(\zeta)}(\zeta)} \text { and } \varphi_{*}(\zeta):=\frac{\varphi(\zeta)}{\left|h_{v(\zeta)}(\zeta)\right|}
$$

are measurable with respect to the logarithmic capacity. 
The logarithmic capacity of a set coincides with its transfinite diameter (see, e.g., point 110 in [15]). Moreover, quasiconformal mappings are Hölder continuous on compacta (see, e.g., Theorem II.4.3 in [1]). Hence the mappings $h$ and $h^{-1}$ transform sets of logarithmic capacity zero on $\partial D$ into sets of logarithmic capacity zero on $\partial D^{*}$, where $D^{*}:=h(D)$, and vice versa. Further, the functions $N:=\left.\nu_{*} \circ h^{-1}\right|_{\partial D^{*}}$ and $\Phi:=\left.\varphi_{*} \circ h^{-1}\right|_{\partial D^{*}}$ are measurable with respect to the logarithmic capacity. Indeed, a measurable set with respect to the logarithmic capacity is transformed under the mappings $h$ and $h^{-1}$ into measurable sets with respect to the logarithmic capacity. Really, such a set can be represented as the union of a sigma-compactum and a set of logarithmic capacity zero. On the other hand, the compacta are transformed under continuous mappings into compacta, and the compacta are measurable with respect to the logarithmic capacity.

Recall that the distortion of angles under quasiconformal mappings $h$ and $h^{-1}$ is bounded (see, e.g., [1]). Thus, nontangential paths to $\partial D$ are transformed into nontangential paths to $\partial D^{*}$ for a.e. $\zeta \in \partial D$ with respect to the logarithmic capacity and inversely. By Theorem 3 in [3], one can find a harmonic function $U: D^{*} \rightarrow \mathbb{R}$ that has the angular limit

$$
\lim _{w \rightarrow \xi} \frac{\partial U}{\partial \mathcal{N}}(w)=\Phi(\xi) \text { q.e. on } \partial D^{*} \text {. }
$$

Moreover, one can find a harmonic function $V$ in the simply connected domain $D^{*}$ such that $F=U+i V$ is an analytic function and, thus, $u:=\operatorname{Re} f=U \circ h$, where $f:=F \circ h$, is a desired $A$-harmonic function in Theorem 2, because $f$ is a regular solution of the corresponding Beltrami equation (1) and

$$
u_{v}=\left\langle\nabla U \circ h, h_{v}\right\rangle=\left\langle v_{*} \nabla U \circ h, v_{*} h_{v}\right\rangle=\left\langle\frac{\partial U}{\partial \mathcal{N}} \circ h, v_{*} h_{v}\right\rangle=\frac{\partial U}{\partial \mathcal{N}} \circ h \operatorname{Re}\left(v_{*} h_{v}\right) .
$$

The following statement concerning the Neumann problem for $A$-harmonic functions is a partial case of Theorem 2.

Corollary 2. Let $D$ be a domain in $\mathbb{C}$ with the quasihyperbolic boundary condition, and let $\partial D$ have a tangent q.e. Suppose that $A(z), z \in D$, is a matrix function in the class $\mathcal{B} \cap C^{\alpha}, \alpha \in(0,1)$, the interior unit normal $n=n(\zeta)$ to $\partial D$ is in the class $\mathcal{C B V}$, and $\varphi: \partial D \rightarrow \mathbb{R}$ is measurable with respect to the logarithmic capacity. Then there is an A-harmonic function $u: D \rightarrow \mathbb{R}$ of the class $C^{1+\alpha}$ such that there exist q.e. on $\partial D$ :

1) the finite limit along the normal $n(\zeta) \quad u(\zeta):=\lim _{z \rightarrow \zeta} u(z)$;

2) the normal derivative $\quad \frac{\partial u}{\partial n}(\zeta):=\lim _{t \rightarrow 0} \frac{u(\zeta+t n)-u(\zeta)}{t}=\varphi(\zeta)$;

3) the angular limit $\lim _{z \rightarrow \varsigma} \frac{\partial u}{\partial n}(z)=\frac{\partial u}{\partial n}(\zeta)$.

This work was partially supported by grants of the Ministry of Education and Science of Ukraine, the project number is $0119 U 100421$. 


\section{REFERENCES}

1. Lehto O. \& Virtanen, K. J. (1973). Quasiconformal mappings in the plane. Berlin, Heidelberg: Springer.

2. Efimushkin, A. S. \& Ryazanov, V. I. (2015). On the Riemann-Hilbert problem for the Beltrami equations in quasidisks. J. Math. Sci., 211, No. 5, pp. 646-659.

3. Gutlyanskii, V., Ryazanov, V., Yakubov, E. \& Yefimushkin, A. (2019). On the Hilbert problem for analytic functions in quasihyperbolic domains. Dopov. Nac. acad. nauk Ukr., No. 2, pp. 23-30. doi: https://doi. org/10.15407/dopovidi2019.02.023

4. Gehring, F. W. \& Palka, B. P.(1976). Quasiconformally homogeneous domains. J. Anal. Math., 30, pp. 172-199.

5. Gehring, F. W. \& Martio, O. (1985). Lipschitz classes and quasiconformal mappings. Ann. Acad. Sci. Fenn. Ser. A. I. Math., 10, pp. 203-219.

6. Becker, J. \& Pommerenke, Ch. (1982). Hölder continuity of conformal mappings and nonquasiconformal Jordan curves. Comment. Math. Helv., 57, No. 2, pp. 221-225.

7. Astala, K. \& Koskela, P. (1991). Quasiconformal mappings and global integrability of the derivative. J. Anal. Math., 57, pp. 203-220.

8. Ladyzhenskaya, O. A. \& Ural'tseva, N. N. (1964). Linear and quasilinear elliptic equations. New York, London: Academic Press.

9. Gehring, F. W. \& Martio, O. (1985). Quasiextremal distance domains and extension of quasiconformal mappings. J. Anal. Math., 45, pp. 181-206.

10. Koosis, P. (1998). Introduction to $H_{\mathrm{p}}$ spaces, Cambridge Tracts in Mathematics. (Vol. 115). Cambridge: Cambridge Univ. Press.

11. Goluzin, G. M. (1969). Geometric theory of functions of a complex variable. Translations of Mathematical Monographs. (Vol. 26). Providence, R.I.: American Mathematical Society.

12. Astala, K., Iwaniec, T. \& Martin, G. (2009). Elliptic partial differential equations and quasiconformal mappings in the plane. Princeton Mathematical Series. (Vol. 48). Princeton: Princeton Univ. Press.

13. Gutlyanskii, V., Ryazanov, V. \& Yefimushkin, A. (2016). On the boundary-value problems for quasiconformal functions in the plane. J. Math. Sci., 214, No. 2, pp. 200-219.

14. Iwaniec, T. (1979). Regularity of solutions of certain degenerate elliptic systems of equations that realize quasiconformal mappings in $n$-dimensional space. Differential and integral equations. Boundary value problems. Tbilisi: Tbilis. Gos. Univ., pp. 97-111.

15. Nevanlinna, R. (1944). Eindeutige analytische Funktionen. Michigan: Ann Arbor.

Received 26.12.2018

В.Я. Гутлянський ${ }^{1}$, В.І. Рязанов ${ }^{1,2}$, Е. Якубов ${ }^{3}$, А.С. Ефімушкін ${ }^{1}$

${ }^{1}$ Інститут прикладної математики і механіки НАН України, Слов'янськ

2 Черкаський національний університет ім. Богдана Хмельницького

${ }^{3}$ Холонський інститут технологій, Ізраїль

E-mail: vgutlyanskii@gmail.com, vl.ryazanov1@gmail.com, yakubov@hit.ac.il, eduardyakubov@gmail.com, a.yefimushkin@gmail.com

\section{ПРО КРАЙОВІ ЗАДАЧІ В ОБЛАСТЯХ БЕЗ (А)-УМОВИ}

Вивчено крайову задачу Гільберта для рівнянь Бельтрамі в жорданових областях, які задовольняють квазігіперболічну крайову умову Герінга-Мартіо, взагалі кажучи, без стандартної (А)-умови ЛадиженськоїУральцевої. 3 припущенням, що коефіцієнти задачі є функціями зліченно-обмеженої варіації і граничні дані є вимірними відносно логарифмічної ємності, доведено існування розв’язків цієї задачі. Як наслідки отримано існування некласичних розв'язків крайових задач Діріхле, Неймана і Пуанкаре для узагальнень рівняння Лапласа в анізотропних і неоднорідних середовищах.

Ключові слова: крайові задачі Гільберта, Діріхле, Неймана і Пуанкаре, рівняния Бельтрамі, квазіконформні функиії, кутова граниия, квазігіперболічна крайова умова, логарифмічна ємність. 
В.Я. Гутлянский ${ }^{1}$ В.И. Рязанов ${ }^{1,2}$, Э. Якубов ${ }^{3}$, А.С. Ефимушкин ${ }^{1}$

${ }^{1}$ Институт прикладной математики и механики НАН Украины, Славянск

${ }^{2}$ Черкасский национальный университет им. Богдана Хмельницкого

${ }^{3}$ Холонский институт технологий, Израиль

E-mail: vgutlyanskii@gmail.com, vl.ryazanov1@gmail.com, yakubov@hit.ac.il, eduardyakubov@gmail.com, a.yefimushkin@gmail.com

\section{О КРАЕВЫХ ЗАДАЧАХ В ОБЛАСТЯХ БЕЗ (А)-УСЛОВИЯ}

Изучена краевая задача Гильберта для уравнений Бельтрами в жордановых областях, удовлетворяющих квазигиперболическому краевому условию Геринга-Мартио, вообще говоря, без стандартного (А)-условия Ладыженской-Уральцевой. С предположением, что коэффициенты задачи являются функциями счетно-ограниченной вариации, а граничные данные измеримы относительно логарифмической емкости, доказано существование решений этой задачи. В качестве следствий получено существование неклассических решений краевых задач Дирихле, Неймана и Пуанкаре для обобщений уравнения Лапласа в анизотропных и неоднородных средах.

Ключевые слова: краевые задачи Гильберта, Дирихле, Неймана и Пуанкаре, уравнения Бельтрами, квазиконформные функиии, угловой предел, квазигиперболическое краевое условие, логарифмическая емкость. 